Nonlin. Processes Geophys., 20, 19-23, 2013

www.nonlin-processes-geophys.net/20/19/2013/

doi:10.5194/npg-20-19-2013

(C) Author(s) 2013. CC Attribution 3.0 License.

\title{
Time scale of the largest imaginable magnetic storm
}

\author{
V. M. Vasyliūnas \\ Max-Planck-Institut für Sonnensystemforschung, 37191 Katlenburg-Lindau, Germany \\ Correspondence to: V. M. Vasyliūnas (vasyliunas@mps.mpg.de)
}

Received: 29 April 2012 - Revised: 19 November 2012 - Accepted: 29 December 2012 - Published: 8 January 2013

\begin{abstract}
The depression of the horizontal magnetic field at Earth's equator for the largest imaginable magnetic storm has been estimated (Vasyliūnas, 2011a) as -Dst $2500 \mathrm{nT}$, from the assumption that the total pressure in the magnetosphere (plasma plus magnetic field perturbation) is limited, in order of magnitude, by the minimum pressure of Earth's dipole field at the location of each flux tube. The obvious related question is how long it would take the solar wind to supply the energy content of this largest storm. The maximum rate of energy input from the solar wind to the magnetosphere can be evaluated on the basis either of magnetotail stress balance or of polar cap potential saturation, giving an estimate of the time required to build up the largest storm, which (for solar-wind and magnetospheric parameter values typical of observed superstorms) is roughly between $\sim 2$ and $\sim 6 \mathrm{~h}$.
\end{abstract}

\section{Introduction}

The current interest in extreme space weather events such as the occasional geomagnetic "superstorms" (e.g. Tsurutani et al., 1992, 2008; Dal Lago et al., 2006; Echer et al., 2008; Gonzalez et al., 2011) and in particular the historic "Carrington" storm of September 1859 (Tsurutani et al., 2003) has led to the concept of the largest imaginable magnetic storm: "the largest depression of the geomagnetic field that could possibly occur" as the result of interaction with the solar wind, in the formulation by Vasyliūnas (2011a), who estimated the maximum depression as $-\mathrm{Dst} \sim 2500 \mathrm{nT}$ by postulating some (unspecified) super-effective plasma transport process that can enhance the total pressure up to maximum value everywhere in the magnetosphere. The purpose of this paper is to seek an answer to the question mentioned but left open by Vasyliūnas (2011a): how much time would be needed to build up the largest imaginable storm by supplying energy from the solar wind?

\section{Energy content in the magnetosphere}

The upper limit on -Dst was derived by assuming the energy content $U_{K}$ of the magnetosphere to be limited ultimately by compression against the Earth's magnetic dipole field and applying the Dessler-Parker-Sckopke theorem, to obtain

$\mu \cdot \mathbf{b}(0) \simeq 2 U_{K}<\frac{2}{27} \frac{\mu^{2}}{R_{\mathrm{E}}^{3}}$

( $\mu=$ dipole moment, $R_{\mathrm{E}}=$ Earth radius). The calculated maximum $2 U_{K}$ is simply a fraction $(\sim 2 / 27)$ of the energy in the geomagnetic field above the Earth's surface, and -Dst is the corresponding fraction of the equatorial surface geomagnetic field $B_{\mathrm{E}}$ (the origin of the factor $2 / 27$ is explained in Vasyliūnas, 2011a). To first approximation, these values are independent of solar-wind parameters; the lowest-order corrections to Eq. (1) are $\mathrm{O}\left(R_{\mathrm{E}} / R_{\mathrm{CF}}\right)^{3}$, where $R_{\mathrm{CF}}$ is the Chapman-Ferraro distance defined by

$R_{\mathrm{CF}}{ }^{3} \equiv \frac{2 \mu}{\sqrt{8 \pi \rho V^{2}}} \simeq\left(7.3 R_{\mathrm{E}}\right)^{3}\left(\frac{10 \mathrm{nPa}}{\rho V^{2}}\right)^{1 / 2}$

with $\rho=$ solar-wind mass density and $V=$ bulk velocity (Gaussian units are used throughout this paper). The question investigated in this paper can now be stated quantitatively: what is the time $\tau$ required to supply the energy content

$U_{K} \sim \frac{1}{27} \frac{\mu^{2}}{R R_{\mathrm{E}}^{3}}=9.2 \times 10^{16} \mathrm{~J}$

to the magnetosphere from the solar wind?

\section{Energy input rate from the solar wind}

The time scale $\tau$ can be estimated as

$\tau \simeq \frac{U_{K}}{\mathcal{P}}$ 
where $U_{K}$ is the energy content given by Eq. (1) and $\mathcal{P}$ is the energy input rate from solar wind into magnetosphere. Unlike $U_{K}$, which to first approximation depends on terrestrial parameters only, $\mathcal{P}$ depends sensitively on solar-wind, magnetospheric, and terrestrial parameters. A scaling law for $\mathcal{P}$ can be derived by dimensional analysis (Vasyliūnas et al., 1982; Vasyliūnas, 2009):

$$
\begin{aligned}
\mathcal{P} & =\frac{1}{2} \rho V^{3} \pi R_{\mathrm{CF}^{2}}{ }^{2} \Psi \\
& \simeq 3.4 \times 10^{13} \mathrm{~W}\left[\frac{V}{10^{3} \mathrm{~km} \mathrm{~s}^{-1}}\right]\left[\frac{\rho V^{2}}{10 \mathrm{nPa}}\right]^{2 / 3} \Psi
\end{aligned}
$$

where

$$
\Psi=\Psi\left(\frac{B}{\sqrt{4 \pi \rho V^{2}}}, \frac{4 \pi \Sigma_{\mathrm{P}} V}{c^{2}}, \theta\right)
$$

is a dimensionless function of dimensionless arguments ( $B=$ magnitude and $\theta=$ direction of interplanetary magnetic field, $\Sigma_{\mathrm{P}}=$ Pedersen conductance of ionosphere). $\Psi$ represents the magnetospheric energy input normalized to the flow of solar-wind kinetic energy through a crosssectional area of radius $R_{\mathrm{CF}}$; equivalently, $\pi R_{\mathrm{CF}}{ }^{2} \Psi$ is the effective cross-section of the magnetosphere for extracting energy from solar-wind flow.

For evaluating the time scale of the largest imaginable storm, the maximum value that $\Psi$ may assume and its dependence on the dimensionless parameters of Eq. (6) needs to be estimated. The fact that intense storms occur when the interplanetary magnetic field is directed predominantly southward for prolonged periods (Gonzalez et al., 1994) defines the condition on $\theta$ required for maximum $\Psi$. The dependence on the other parameters and the numerical value of $\Psi_{\max }$ can be roughly estimated, by a combination of theoretical and empirical arguments, in several ways. Magnetotail stress balance (Appendix A) gives

$\Psi_{\max } \sim \frac{1}{4}$,

a constant, independent of other parameters. Polar cap potential saturation (Appendix B) gives

$\Psi_{\max } \sim \frac{1}{7}\left[\frac{10^{3} \mathrm{~km} \mathrm{~s}^{-1}}{V}\right]$

from the observed saturation level, or

$\Psi_{\max } \sim\left(\frac{1}{6}\right.$ to $\left.\frac{1}{10}\right)\left[\frac{10^{3} \mathrm{~km} \mathrm{~s}^{-1}}{V}\right]\left[\frac{10 \mathrm{mho}}{\Sigma_{\mathrm{P}}}\right]$

from theoretical models. Since these are estimates of the maximum values, expected under extreme conditions, it is not surprising that they are somewhat larger than the values (usually $\sim 0.05$ to 0.1 ) found in empirical determinations (e.g. Gonzalez et al., 1989; Gonzalez, 1990; Weiss et al., 1992; Koskinen and Tanskanen, 2002, and many others).

\section{Estimate of time scale}

Combining Eqs. (1), (4), and (5) gives the time scale required to build up the largest imaginable magnetic storm as

$\tau \simeq \frac{1}{27} \frac{\mu^{2}}{R_{\mathrm{E}}^{3}}\left[\frac{1}{2} \rho V^{3} \pi R_{\mathrm{CF}}^{2} \Psi_{\max }\right]^{-1}$

or, noting that $\mu^{2} \equiv\left(B_{\mathrm{E}} R_{\mathrm{E}}{ }^{3}\right)^{2}=2 \pi R_{\mathrm{CF}}^{6} \rho V^{2}$,

$$
\begin{aligned}
\tau \simeq \frac{4}{27} & \frac{1}{\Psi_{\max }}\left(\frac{R_{\mathrm{E}}}{V}\right)\left(\frac{R_{\mathrm{CF}}}{R_{\mathrm{E}}}\right)^{4} \\
\simeq & \frac{0.373}{\Psi_{\max }}\left(\frac{R_{\mathrm{E}}}{V}\right)\left(\frac{B_{\mathrm{E}}^{2}}{8 \pi \rho V^{2}}\right)^{2 / 3} .
\end{aligned}
$$

With numerical values for $\Psi_{\max }$ from Sect. 3, the estimated time scale becomes the following: from Eq. (7) (magnetotail stress balance)

$\tau \simeq\left[\frac{10^{3} \mathrm{~km} \mathrm{~s}^{-1}}{V}\right]\left[\frac{10 \mathrm{nPa}}{\rho V^{2}}\right]^{2 / 3} 140 \mathrm{~min}$,

Eq. (8) (polar cap potential saturation, empirical)

$\tau \simeq\left[\frac{10 \mathrm{nPa}}{\rho V^{2}}\right]^{2 / 3} 240 \mathrm{~min}$,

and Eq. (9) (polar cap potential saturation, theoretical)

$\tau \simeq\left[\frac{10 \mathrm{nPa}}{\rho V^{2}}\right]^{2 / 3}\left[\frac{\Sigma_{\mathrm{P}}}{10 \mathrm{mho}}\right] 210-350 \mathrm{~min}$.

For solar-wind parameters typical of observed very large storms, the time scale is about 2 to $6 \mathrm{~h}$ (shorter if the solarwind dynamic pressure significantly exceeds $10 \mathrm{nPa}$ ).

\section{Conclusions}

The rate of energy input from the solar wind, under conditions expected for extreme storms, appears to be sufficient (albeit not by an overwhelming margin) to supply the energy content of the largest imaginable magnetic storm within a reasonable time (several hours).

Not considered here is one remaining open question concerning the largest imaginable magnetic storm: can the postulated super-effective transport process, capable of filling the magnetosphere with plasma pressure up to the maximum possible value everywhere, actually occur, and if so, under what conditions?

\section{Appendix A}

\section{Energy input estimated from magnetotail stress balance}

The net magnetic tension force in the magnetotail (Siscoe, 1966), exerted ultimately on the Earth, is applied from the 
solar wind, predominantly along open magnetic field lines. The work done by solar-wind plasma flow against this force is the primary source of energy supply to the magnetosphere, as proposed by Siscoe and Cummings (1969) (see also Siscoe and Crooker, 1974). The quantitative stress-balance relation is (Siscoe, 1966; Vasyliūnas, 1987; Vasyliūnas, 2009)

$$
\frac{B_{T}^{2}}{8 \pi}(1-\delta) A_{T}-S \Delta V_{x} \simeq 0
$$

where $B_{T}=$ magnetotail (lobe) field, $A_{T}=$ cross-sectional area of magnetotail at its Earthward boundary, $S=$ total plasma mass flow through region of interaction with solar wind (e.g. plasma mantle), and $\Delta V_{x}=$ average slowdown of antisunward flow; $\delta=$ correction term for plasma sheet and for tail flaring (Siscoe, 1972a,b; Carovillano and Siscoe, 1973; Vasyliūnas, 1987). The corresponding energy input rate is, quite generally (e.g. Vasyliūnas, 2009, 2010), $\mathcal{P}=S V \Delta V_{x}$, which combined with Eq. (A1) gives

$\mathcal{P}=S V \Delta V_{x}=\frac{1}{2} \rho V^{3} A_{T}(1-\delta) \frac{2 B_{T}{ }^{2}}{8 \pi \rho V^{2}}$

or, comparing with Eq. (5),

$\Psi \simeq \frac{2 A_{T}(1-\delta)}{\pi R_{\mathrm{CF}}^{2}} \frac{B_{T}^{2}}{8 \pi \rho V^{2}}$.

Normally, the bulk of the energy input given by Eq. (A2) goes down the magnetotail and only a limited fraction goes into the inner magnetosphere (Vasyliūnas, 2010, 2011b). To obtain an estimate for $\Psi_{\max }$, I assume that (1) under appropriate conditions (and as a property of the postulated super-effective transport process that produces the largest imaginable magnetic storm), the energy input of Eq. (A2) can go predominantly into building up the energy content of the inner magnetosphere, (2) the magnetotail parameters in Eq. (A3) retain, within on order of magnitude, their typical empirically estimated values $A_{T} / \pi R_{\mathrm{CF}}{ }^{2} \sim 4$, $B_{T} / \sqrt{8 \pi \rho V^{2}} \sim 1 / 3$, and $\delta \sim 0.5$ to 0.7 (Carovillano and Siscoe, 1973; Vasyliūnas, 1987). Assumption (2) appears reasonable for the first two parameters, which are determined largely by geometry and by pressure balance; less so perhaps for $\delta$, which depends appreciably on the ratio (plasma sheet cross-sectional area) $/ A_{T}$ (Carovillano and Siscoe, 1973) and hence on the ratio of closed to open magnetic flux in the magnetotail - but the increased amount of open flux expected for intense storms should lead rather to a decrease of $\delta$ compared to the average value quoted above. With these two assumptions, Eq. (A3) then gives the estimate for $\Psi_{\max }$ in Eq. (7).

\section{Appendix B}

\section{Energy input estimated from polar cap potential saturation}

An intuitively obvious estimate of the energy input rate is

$\mathcal{P}=\Phi_{\mathrm{PC}} I_{\mathrm{M}}$

the product of $\Phi_{\mathrm{PC}}$, the (so-called) cross-polar-cap potential (actually the potential across much of the magnetosphere and magnetotail) times $I_{\mathrm{M}}$, the total current flowing across this potential in the magnetosphere (not just in the polar cap). From dimensional analysis

$$
\begin{aligned}
\Phi_{\mathrm{PC}} & =\frac{1}{c} V B R_{\mathrm{CF}} \Psi_{\mathrm{PC}}, \\
I_{\mathrm{M}} & =\frac{c}{4 \pi} \sqrt{8 \pi \rho V^{2}} R_{\mathrm{CF}} \Psi_{I},
\end{aligned}
$$

where $\Psi_{\mathrm{PC}}$ and $\Psi_{I}$ are dimensionless functions of the same dimensionless variables as $\Psi$ in Eq. (6). Comparison of Eqs. (5) and (B1) gives the relation

$\Psi=\Psi_{\mathrm{PC}} \Psi_{I} \frac{B}{\sqrt{4 \pi \rho V^{2}}} \frac{2 \sqrt{2}}{\pi}$.

There are diverse theoretical models for $\Phi_{\mathrm{PC}}$ and $\Psi_{I}$ (e.g. Gonzalez and Mozer, 1974; Kan and Lee, 1979; Kan et al., 1980, and others), but more relevant for the purposes of this paper are the upper limits on both quantities. The total magnetospheric current is limited by global stress balance between magnetic field and solar-wind dynamic pressure and by the size of magnetosphere. The limiting current $I_{\mathrm{M}}$ calculated from a simple hemispherical model of the magnetopause, combined with the Newtonian approximation for the exterior pressure, is given by Eq. (B3) with $\Psi_{I} \leq 4$; numerically,

$I_{\mathrm{M}} \leq 2.35 \times 10^{7} \mathrm{~A}\left[\frac{\rho V^{2}}{10 \mathrm{nPa}}\right]^{1 / 3}$.

The transition, during intense storms, from pressure balance to dissipative tangential stress is discussed by Siscoe (2006, 2011).

The cross-polar-cap potential $\Phi_{\mathrm{PC}}$ is observed to become saturated, i.e. independent of $V B$ (for fixed solar-wind dynamic pressure $\rho V^{2}$ ) when $V B$ becomes sufficiently large; see review by Shepherd (2007) and references therein. Equation (B2) can be rewritten as

$\Phi_{\mathrm{PC}}=\left[\frac{1}{c} \sqrt{4 \pi \rho V^{2}} R_{\mathrm{CF}}\right]\left[V_{\mathrm{A}} \Psi_{\mathrm{PC}}\right]$

$\left(V_{\mathrm{A}}=B / \sqrt{4 \pi \rho}=\right.$ Alfvén speed in the solar wind), where all the factors that depend on solar-wind dynamic pressure 
(only) have been collected into the first pair of brackets (easily shown to vary as $\left.\left[\rho V^{2}\right]^{1 / 3}\right)$; if saturation occurs, the quantity in the second pair of brackets $\left(V_{\mathrm{A}} \Psi_{\mathrm{PC}}\right)$ must therefore be independent of $V B$. This can be used to infer $\Psi_{\max }$ in two different ways.

Empirically, $V_{\mathrm{A}} \Psi_{\mathrm{PC}}$ can be treated (in the saturation regime) as simply a number to be determined by a fit to observations; this provides a limiting potential which, together with the limiting current, allows $\Psi_{\max }$ to be estimated without further appeal to dimensional analysis. Hairston et al. (2005) report saturation values $\Phi_{\mathrm{PC}} \sim 200 \mathrm{kV}$ during large storms, when $\rho V^{2} \sim 10 \mathrm{nPa}$. Multiplying this $\Phi_{\mathrm{PC}}$ by $I_{\mathrm{M}}$ from Eq. (B5) and comparing with the numerical values in Eq. (5) yields $\Psi_{\max }$ given in Eq. (8).

Theoretically, $V_{\mathrm{A}} \Psi_{\mathrm{PC}}$ can be independent of $V B$ only if $\Psi_{\mathrm{PC}}$ (a dimensionless function of dimensionless arguments) has the form

$\Psi_{\mathrm{PC}} \simeq \Psi_{0} \frac{\sqrt{4 \pi \rho V^{2}}}{B} \frac{c^{2}}{4 \pi \Sigma_{\mathrm{P}} V}=\Psi_{0} \frac{c^{2}}{4 \pi \Sigma_{\mathrm{P}} V_{\mathrm{A}}}$

where $\Psi_{0}=$ constant. The additional restriction $\Psi_{\mathrm{PC}}<1$ (from the observed fact that $\Phi_{\mathrm{PC}}$ is at most a fraction of the solar-wind potential across the magnetosphere) implies that Eq. (B7) must be a limiting form, valid only for $4 \pi \Sigma_{\mathrm{P}} V_{\mathrm{A}} / c^{2} \gg 1$. Given the premise that polar cap potential saturation occurs, simple dimensional analysis thus suffices to establish Eq. (B7) and the corresponding expression for the saturated potential,

$$
\begin{aligned}
\Phi_{\mathrm{PC}} & \simeq \Psi_{0} R_{\mathrm{CF}} \sqrt{4 \pi \rho V^{2}} \frac{c}{4 \pi \Sigma_{\mathrm{P}}} \\
& \simeq 416 \mathrm{kV} \Psi_{0}\left[\frac{\rho V^{2}}{10 \mathrm{nPa}}\right]^{1 / 3}\left[\frac{10 \mathrm{mho}}{\Sigma_{\mathrm{P}}}\right],
\end{aligned}
$$

together with the consequence that saturation (at least in the strict sense of complete independence from $V B$ ) is possible only if the physical process involves a (sufficiently large) $\Sigma_{\mathrm{P}}$. Dimensional analysis does not require any additional assumptions, but neither does it provide any further information about the physical process, and it does not determine the value of $\Psi_{0}$. Specific physical models for polar cap potential saturation have been proposed by Hill et al. (1976), Siscoe et al. (2002), and Kivelson and Ridley (2008); they all predict a dependence of the potential on $\Sigma_{\mathrm{P}}$ that, when expressed in dimensionless terms, gives

$\Psi_{\mathrm{PC}}=\frac{\Psi_{0}}{\Psi_{0}+4 \pi \Sigma_{\mathrm{P}} V_{\mathrm{A}} / c^{2}}$

which reduces to Eq. (B7) in the limit $4 \pi \Sigma_{\mathrm{P}} V_{\mathrm{A}} / c^{2} \gg 1$. Hill et al. (1976) simply assume $\Psi_{0}=1$; the others calculate $\Psi_{0}=0.608$ (Siscoe et al., 2002, from a semi-empirical fit to data/simulation) or $\Psi_{0}=0.344$ (Kivelson and Ridley, 2008, from theory). Inserting the limiting value Eq. (B7) into Eq. (B4) gives
$\Psi_{\max } \simeq \Psi_{0} \frac{8 \sqrt{2}}{\pi} \frac{c^{2}}{4 \pi \Sigma_{\mathrm{P}} V}$.

With the above calculated values for $\Psi_{0}$, Eq. (B10) gives the estimates for $\Psi_{\max }$ in Eq. (9). (Rigorous application of Eq. (B9) implies the substitution

$\left[\frac{10 \mathrm{mho}}{\Sigma_{\mathrm{P}}}\right] \longrightarrow\left[\frac{\Sigma_{\mathrm{P}}}{10 \mathrm{mho}}+\Psi_{0} \frac{80 \mathrm{~km} \mathrm{~s}^{-1}}{V_{\mathrm{A}}}\right]^{-1}$

in Eqs. (9), (15), and (B8), showing how upper limits that depend on ionospheric conductance can be produced by strong interplanetary magnetic fields typical of intense storms).

The method of estimating the energy input rate used here in Appendix B is fundamentally equivalent to that in Appendix A (Vasyliūnas et al., 1982) but yields lower values of $\Psi_{\max }$ (hence longer time scales) because it takes into account additional constraints imposed by the ionosphere, manifested in the phenomenon of polar cap potential saturation.

Acknowledgements. This work was motivated in part by continuing interest and questions from Bruce Tsurutani and Walter Gonzalez. I am grateful to the referees for useful comments that helped clarify some significant aspects.

The service charges for this open access publication have been covered by the Max Planck Society.

Edited by: B. Tsurutani

Reviewed by: two anonymous referees

\section{References}

Carovillano, R. L. and Siscoe, G. L.: Energy and momentum theorems in magnetospheric dynamics, Rev. Geophys. Space Phys., 11, 289-353, 1973.

Dal Lago, A., Gonzalez, W. D., Balmaceda, L. A., Vieira, L. E. A., Echer, E., Guarnieri, F. L., Santos, J., da Silva, M. R., de Lucas, A., Clúa de Gonzalez, A. L., Schwenn, R., and Schuch, N. J.: The 17-22 October (1999) solar-interplanetarygeomagnetic event: Very intense geomagnetic storm associated with a pressure balance between interplanetary coronal mass ejection and a high-speed stream, J. Geophys. Res., 111, A07S14, doi:10.1029/2005JA011394, 2006.

Echer, E., Gonzalez, W. D., and Tsurutani, B. T.: Interplanetary conditions leading to superintense geomagnetic storms (Dst $\leq-250$ nT) during solar cycle 23, Geophys. Res. Lett., 35, L06S03, doi:10.1029/2007GL031755, 2008.

Gonzalez, W. D.: A unified view of solar wind-magnetosphere coupling functions, Planet. Space Sci., 38, 627-632, 1990.

Gonzalez, W. D. and Mozer, F. S.: A quantitative model for the potential resulting from reconnection with an arbitrary interplanetary magnetic field, J. Geophys. Res., 79, 4186-4194, 1974.

Gonzalez, W. D., Tsurutani, B. T., Gonzalez, A. L. C., Smith, E. J., Tang, F., and Akasofu, S.-I.: Solar wind-magnetosphere coupling 
during intense magnetic storms (1978-1979), J. Geophys. Res., 94, 8835-8851, 1989.

Gonzalez, W. D., Joselyn, J. A., Kamide, Y., Kroehl, H. W., Rostoker, G., Tsurutani, B. T., and Vasyliūnas, V. M.: What is a geomagnetic storm?, J. Geophys. Res., 99, 5771-5792, 1994.

Gonzalez, W. D., Echer, E., Tsurutani, B. T., Clúa de Gonzalez, A. L., and Dal Lago, A.: Interplanetary origin of intense, superintense and extreme geomagnetic storms, Space Sci. Rev., 158, 69-89, doi:10.1007/s11214-010-9715-2, 2011.

Hairston, M. R., Drake, K. A., and Skoug, R.: Saturation of the ionospheric polar cap potential during the OctoberNovember 2003 superstorms, J. Geophys. Res., 110, A09S26, doi:10.1029/2004JA010864, 2005.

Hill, T. W., Dessler, A. J., and Wolf, R. A.: Mercury and Mars: The role of ionospheric conductivity in the acceleration of magnetospheric particles, Geophys. Res. Lett., 3, 429-432, 1976.

Kan, J. R. and Lee, L. C.: Energy coupling function and solar windmagnetosphere dynamo, Geophys. Res. Lett, 6, 577-580, 1979.

Kan, J. R., Lee, L. C. and Akasofu, S.-I.: The energy coupling function and the power generated by the solar wind-magnetosphere dynamo, Planet. Space Sci., 28, 823-825, 1980.

Kivelson, M. G. and Ridley, A. J.: Saturation of the polar cap potential: Inference from Alfvén wing arguments, J. Geophys. Res., 113, A05214, doi:10.1029/2007JA012302, 2008.

Koskinen, H. E. J. and Tanskanen, E. I.: Magnetospheric energy budget and the epsilon parameter, J. Geophys. Res., 107, 1415, doi:10.1029/2002JA009283, 2002.

Shepherd, S. G.: Polar cap potential saturation: Observations, theory, and modeling, J. Atmos. Solar-Terr. Phys., 69, 234-248, 2007.

Siscoe, G. L.: A unified treatment of magnetospheric dynamics with applications to magnetic storms, Planet. Space Sci., 14, 947-967, 1966.

Siscoe, G. L.: Consequences of an isotropic static plasma sheet in models of the geomagnetic tail, Planet. Space Sci., 20, 937-953, 1972a.

Siscoe, G. L.: On the plasma sheet contribution to the force balance requirements in the geomagnetic tail, J. Geophys. Res., 77, 6230 6234, 1972b.

Siscoe, G. L.: Global force balance of Region 1 current system, J. Atmos. Sol.-Terr. Phys., 68, 2119-2126, 2006.

Siscoe, G. L.: Aspects of global coherence of magnetospheric behavior, J. Atmos. Sol.-Terr. Phys., 73, 402-419, 2011.

Siscoe, G. L. and Cummings, W. D.: On the cause of geomagnetic bays, Planet. Space Sci., 17 1795-1802, 1969.
Siscoe, G. L. and Crooker, N. U.: A theoretical relation between DST and the solar wind merging electric field, Geophys. Res. Lett., 1, 17-19, 1974.

Siscoe, G. L., Erickson, G. M., Sonnerup, B. U. Ö., Maynard, N. C., Schoendorf, J. A., Siebert, K. D., Weimer, D. R., White, W. W., and Wilson, G. R.: Hill model of transpolar potential saturation: Comparisons with MHD simulations, J. Geophys. Res., 107, 1075, doi:10.1029/2001JA000109, 2002.

Tsurutani, B. T., Gonzalez, W. D., Tang, F., and Lee Y. T.: Great magnetic storms, Geophys. Res. Lett., 19, 73-76, 1992.

Tsurutani, B. T., Gonzalez, W. D., Lakhina, G. S., and Alex, S.: The extreme magnetic storm of 1-2 September 1859, J. Geophys. Res., 108, 1268, doi:10.1029/2002JA009504, 2003.

Tsurutani, B. T., Echer, E., Guarnieri, F. L., and Kozyra, J. U.: CAWSES November 7-8, 2004, superstorm: Complex solar and interplanetary features in the post-solar maximum phase, Geophys. Res. Lett., 35, L06S05, doi:10.1029/2007GL031473, 2008.

Vasyliūnas, V. M.: Contribution to "Dialog on the relative roles of reconnection and the "viscous" interaction in providing solarwind energy to the magnetosphere," in: Magnetotail Physics, edited by: Lui, A. T. Y., 411-412, The Johns Hopkins University Press, Baltimore, 1987.

Vasyliūnas, V. M.: The mechanical advantage of the magnetosphere: solar-wind-related forces in the magnetosphereionosphere-Earth system, Ann. Geophys., 25, 255-269, doi:10.5194/angeo-25-255-2007, 2007.

Vasyliūnas, V. M.: Fundamentals of planetary magnetospheres, in: Heliophysics: Plasma Physics of the Local Cosmos, edited by: Schriver, C. J. and Siscoe, G. L., Cambridge University Press, NY, 256-294, 2009.

Vasyliūnas, V. M.: Energy conversion in planetary magnetospheres, in: Heliophysics: Space Storms and Radiation: Causes and Effects, edited by: Schriver, C. J. and Siscoe, G. L., Cambridge University Press, NY, 263-291, 2010.

Vasyliūnas, V. M.: The largest imaginable magnetic storm, J. Atmos. Solar-Terr. Phys. 73, 1444-1446, 2011a.

Vasyliūnas, V. M.: Physics of magnetospheric variability, Space Sci. Rev., 158, 91-118, doi:10.1007/s11214-010-9696-1, $2011 \mathrm{~b}$.

Vasyliūnas, V. M., Kan, J. R., Siscoe, G. L., and Akasofu, S.I.: Scaling relations governing magnetospheric energy transfer, Planet. Space Sci., 30, 359-365, 1982.

Weiss, L. A., Reiff, P. H., Moses, J. J., Heelis, R. A., and Moore, D. B.: Energy dissipation in substorms, in Substorms 1, edited by Mattock, C., 309-317, Eur. Space. Agency Spec. Publ. ESA SP-335, Noordwijk, Netherlands, 1992. 